statement generally made that there is a thinning of the superficial epidermal layer, and an apparent thickening, from infiltration, of the underlying tunica propria. It is possible to see in this an explanation of the want of resiliency which may increase the difficulty of the passage of food. It is not infrequently stated that superficial glossitis is related to syphilis, possibly from the statement of Goldschmidt (Berlin. klin. Wochens., 1899) that it was present in 47 out of 60 cases of smooth atrophy of the tongue. This cannot be said for the state under consideration, for out of a considerable number of cases during the last few years, the Wassermann reaction was negative except in two-and antispecific treatment in both made not the slightest change. In all these cases the effect of nasal disease may be excluded.

The question may, I think, fairly be raised, What relation do these changes bear to the spasmodic condition? Starck points out ("Fsophagoskopie," p. 134) that spasm almost always occurs as a symptom of an anatomical lesion of the esophagus or other organs (stomach, vertebral column, etc.) ; less frequently can it be regarded as a disease sui generis, a motor neurosis. He further states that as symptom it is found most frequently in malignant disease of the gullet, and he has pointed out that it may often possess the significance of an early symptom. The first appearance of dysphagia in carcinoma often does not occur at the seat of the lesion, but rather at the entrance, more rarely at the outlet, of the gullet. In view of the anatomical changes which I have described in the upper food-passage it is not unreasonable to assume that they may take some part in the production of spasm, and we may see in this an explanation of the relationship of these two conditions.

One further point may be remarked upon, viz. the not infrequent supervention in such cases of malignant disease at the mouth of the gullet. This happens too often to be merely a coincidence. In more cases than one under my observation for a long period this termination has occurred.

To sum up: (1) Spasmodic dysphagia in women is sometimes associated with definite changes in the upper food-passage. If they are not the sole cause of spasm, in all probability they increase the tendency.

(2) That the association produces a more obstinate type of dysphagia both in relation to treatment by dilatation and the effect on the general condition of the patient.

\title{
THE AQUEDUCT OF FALLOPIUS AND FACIAL PARALYSIS.
}

\author{
By Dan McKenzie, M.D.
}

\section{Part I: The Aqueduct of Fallopius. \\ (Continued from p. 247.)}

The figures are as follows:

Thirty-nine bones were available-16 acellular and 23 cellularbelonging to all the seven decades reviewed. The greatest distance in acellular bon es was $5 \mathrm{~mm}$, the least $2 \mathrm{~mm}$., the average $3 \cdot 2 \mathrm{~mm}$.

The greatest distance in the cellular bones was $4 \mathrm{~mm}$., the least $2 \mathrm{~mm}$., the average $3 \mathrm{~mm}$. 
There is so little difference, therefore, on the whole between the two varieties in this particular measurement, that for practical purposes it may be ignored. But, such as it is, it indicates a tendency for the Fallopian canal to lie nearer the meatus in cellular bones, as if the development of the cells pushed the Fallopian canal forward.

A similar comparison between acellular and cellular bones was effected with regard to the distance between the groove for the lateral sinus and the posterior meatal wall, with a much more definite result, and one quite in keeping with the usual teaching. Although the point

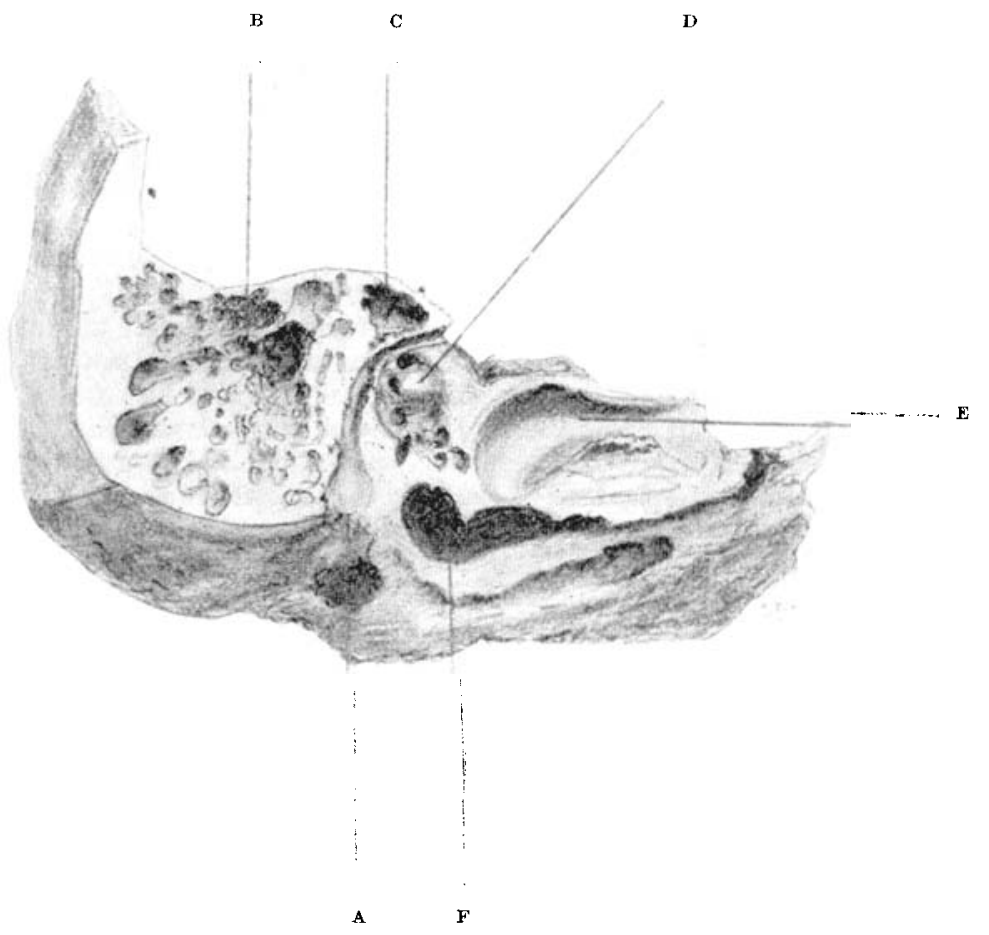

Fig. 11.-Pneumatic mastoid cut to show relationship of cells to the vertical segment of the Fallopian canal. The roof of the tympanum has been removed. A. Stylomastoid foramen. B. Mastoid cells. c. Attic and aditus ad antrum, with tympanic segment of Fallopian aqueduct below. D. Promontory, with the oval window. Note cells leading out of tympanum towards $\mathrm{E}$, the carotid canal, and $\mathrm{F}$, the jugular foramen. (Right temporal, bone 18, female, aged forty-three, slightly reduced, pose rotated )

is one which has no direct bearing upon the anatomy of the Fallopian canal, it is here inserted for its practical interest.

Thirty-four bones were valid for the purpose-15 acellular and 19 cellular. In the former the distances were: Highest, $15 \mathrm{~mm}$.; lowest, $3 \mathrm{~mm}$.; average, $9.7 \mathrm{~mm}$. (The very low reading of $3 \mathrm{~mm}$. was found in a bone of the third decade.) In the cellular bones, on the other hand, the distances were much greater: Highest, $20 \mathrm{~mm}$.; lowest, $10 \mathrm{~mm}$.; average, $13.9 \mathrm{~mm}$. 


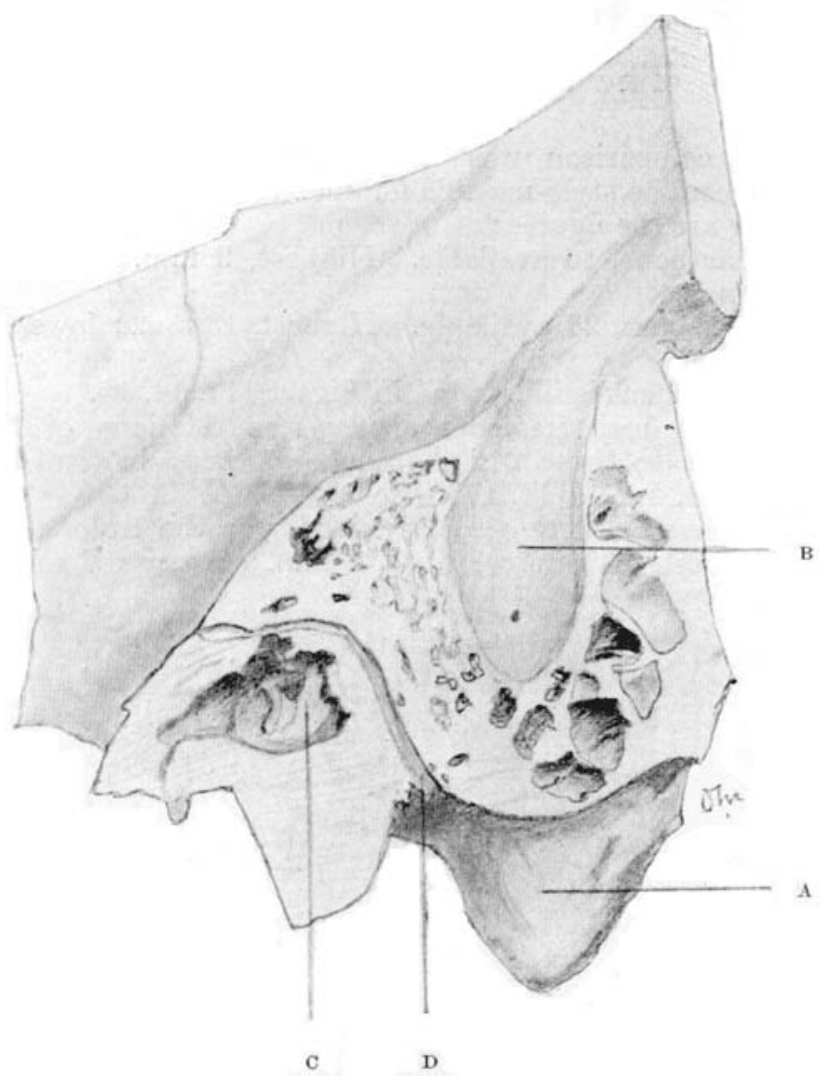

Fig. 12.-A. Mastoid process; B. Lateral sinus groove; and c. Tympanic membrane (with malleus handle) from within, showing relationship to Fallopian canal. D. Stylo-mastoid foramen. (Right temporal, bone 45, male, aged sixty-two, slightly + , pose slightly oblique.)

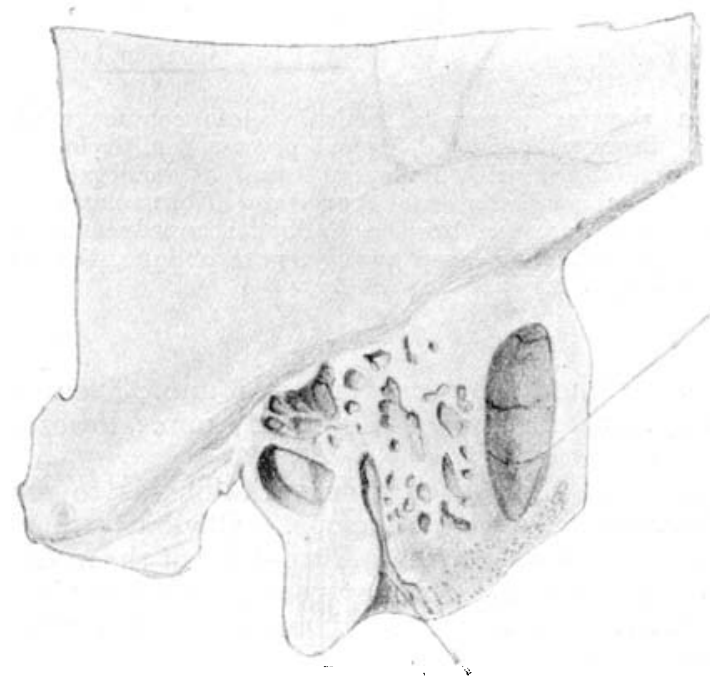

A

Fia. 13.-Temporal bone from within. A. Stylomastoid foramen. B. Lateral sinus groove. The antrum is seen above and behind the external auditory meatus. (Bone 20, right temporal, male, aged fifty-three, reduced one quarter, pose oblique.) 
CThe same comparison was also instituted in the matter of the distance between the stylo-mastoid foramen and the lateral sinus groove. The following are the figures:

In aceilular bone, 16 available. Highest, $9 \mathrm{~mm}$.; lowest, $2 \mathrm{~mm}$.; average, $6.1 \mathrm{~mm}$.

In cellular bones, 23 available. Highest, $13 \mathrm{~mm}$; lowest, $4 \mathrm{~mm}$; average, $7.6 \mathrm{~mm}$.

This measurement is illustrated in Figs. 8, 11, 14, 15.

The following measurements display the relations of the stylomastoid foramen to the tip of the mastoid process in adult bones (see also Figs. 1, 2, 3, 5, 6, 7, 11, 14):

The stylo-mastoid foramen lies anterior to the tip of the mastoid process. Highest, $14 \mathrm{~mm}$; lowest, $2 \mathrm{~mm}$; average, $7 \mathrm{~mm}$. It lies

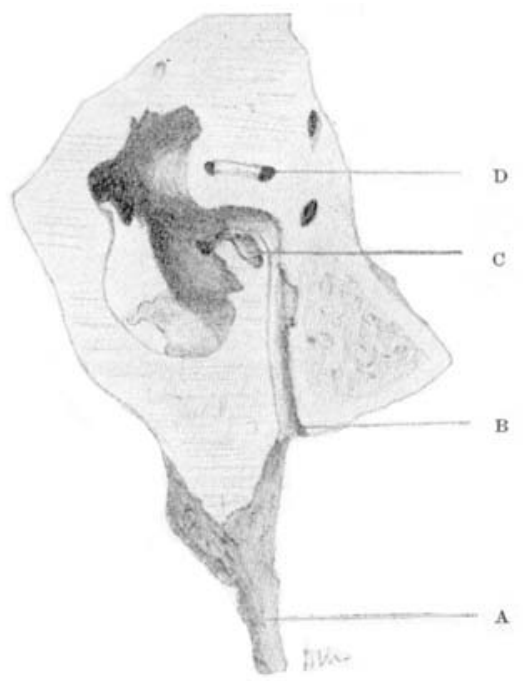

Fis. 14.--Specimen showing pyramidal cells in close contact with the Fallopian canal. From without. A. Styloid process. B. Stylo-mastoid foramen. c. Pyramidal cell with Fallopian canal at bend above and behind it. D. External semicular canal opened up. Note relationship of this canal and its prominence to the Fallopian aqueduct below it. (Bone 28, left temporal, male, aged twenty, pose obliqne, the styloid process being vertical, $\times 2$.)

medial to the tip. Highest, $12 \mathrm{~mm}$; lowest, $3 \mathrm{~mm}$.; average, $7 \mathrm{~mm}$. Its opening lies superior to the tip. Highest, $19 \mathrm{~mm}$.; lowest, $6 \mathrm{~mm}$.; average, $12 \mathrm{~mm}$.

These measurements show such wide variations as to render the taking of any "average" distance fallacious. Obviously they depend upon the size and situation of the mastoid tip-one of the most variable features in the temporal bone. All they permit us to say is that the stylo-mastoid foramen lies anterior, medial, and superior to the tip of the mastoid.

In infantile bones a special note is necessary regarding these data (see later). 


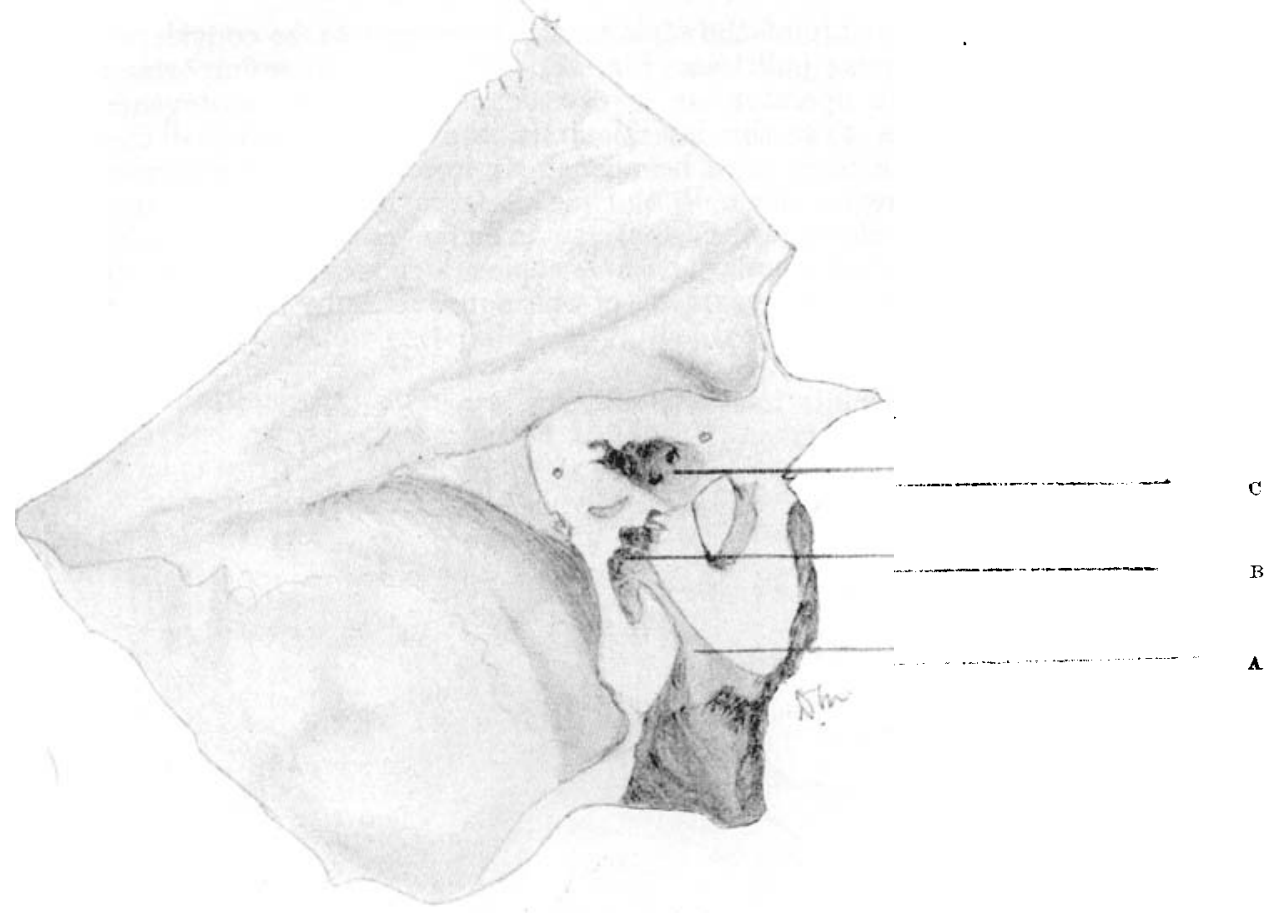

F'ı. 15.-Same temporal bone as Fig. 14. Viewed from within. A. Stylomastoid foramen. B. Cluster of cells continuous with $\mathrm{c}$ in Fig. 14 c. Antrum. Note proximity of lateral sinus groove to cells and canal. (Pose natural, life-size.)

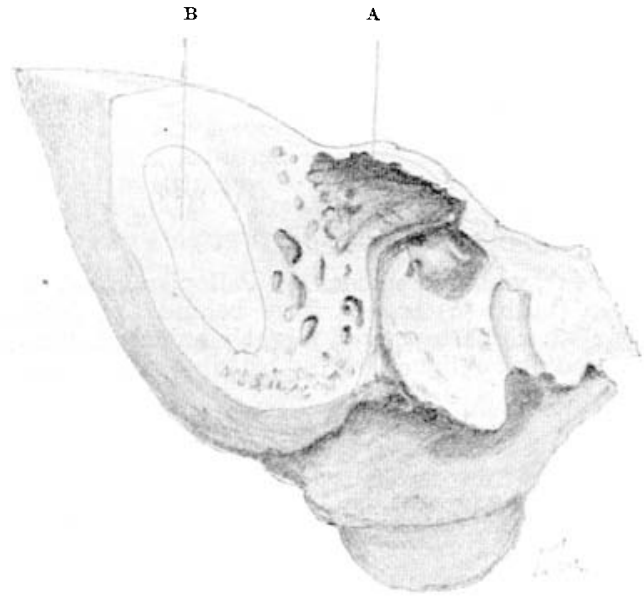

Fia. 16.-Section passes through tympanum, antrum (A), lateral sinus groove (B) and Fallopian canal, the tympanic segment of which has been opened up in its entire length. Note the foramen leading to the geniculate at the junction of the roof and medial wall of the tympanum. (Bone 39, right temporal viewed from without, male, aged fifty-three, pose natural, enlarged by $\frac{1}{4}$.) 
The last relationship of the stylo-mastoid foramen to be considered is that to the jugular bulb (see Fig. 17). This is another important relationship to the operator, as it occasionally happens in otogenic venous thrombosis that the lateral sinus, the jugular bulb and the jugular vein in the neck must be opened up in one continuous gutter. In the effort to reach the bulb the facial nerve in the vertical and at its exit at the stylo-mastoid foramen is endangered, as in a certain number of cases the jugular bulb occupies a position anterior to the plane of the vertical segment of the aqueduct. In the majority

D

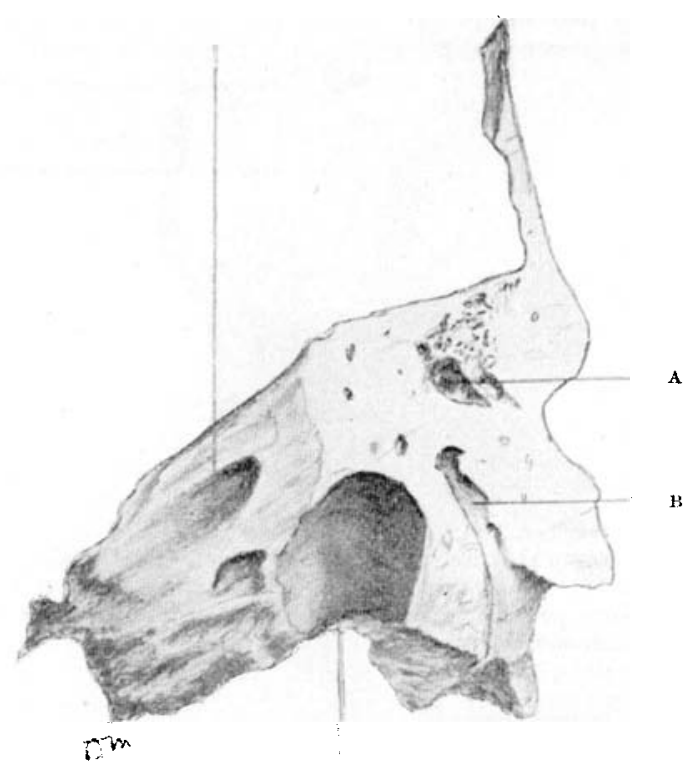

C

Fig. 17.-Antrum (A), vertical segment of Fallopian canal (B) and roof of jugular foramen (bulb) (c) opened from behind. D. Internal auditory meatus. (Bone 43, right temporal, slightly enlarged, male, aged sixtyeight, pose rotated and oblique.)

of instances, however, the bulb could be opened up from behind without endangering the facial nerve.

The numbers are as follows: Forty-three bones in all were available -34 adult, and 9 in the first decade. Of the total 9 only were "inaccessible," and the rest more or less easily accessible from behind without imperilling the facial nerve.

The distances between the stylo-mastoid foramen and the jugular sulcus were (in adult bones): Highest, $11 \mathrm{~mm}$.; lowest, $2 \mathrm{~mm}$.; average, $4.8 \mathrm{~mm}$. And in bones of the first decade: Highest, $7 \mathrm{~mm}$.; lowest, $1 \mathrm{~mm}$. ; average, $4 \cdot 2 \mathrm{~mm}$. 
The recess for the reception of the dome of the jugular bulb shares with the mastoid process the reputation of being extremely variable in size and situation.

In some its situation on the under surface of the petrous is scarcely perceptible. In others, again, it is so great as to encroach upon the middle ear, raising the floor of that cavity to a level that brings the bulb of the jugular vein to a position which would render it liable to be wounded in carrying out the simple every-day procedure of paracentesis for suppuration of the middle ear (Fig. 18). The Cheatle collection contains seven of these large jugular sulci.

The following numbers give the depth of the vertical at its upper end and at the stylo-mastoid foramen from the outer surface of the bone (Fig. 3) :

Depth of upper end of vertical (37 bones valid): Highest, $21 \mathrm{~mm}$.; lowest, $7 \mathrm{~mm}$.; average, $14 \cdot 3 \mathrm{~mm}$.

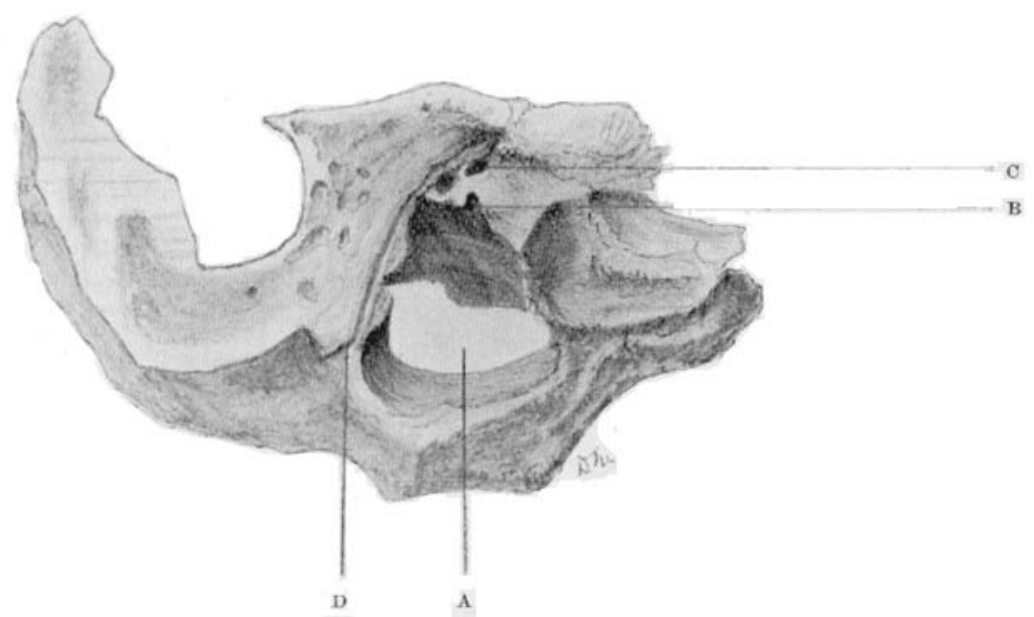

Fia. 18.-Specimen with an enormous jugular bulb (A), which is seen extending up into the tympanum as high as the niche of the round window $(B)$. C. The oval window. D. The stylo-mastoid foramen. Part of the thin bony floor of the tympanum has been removed. (Bone 27, right temporal, slightly enlarged, male, aged sixty-eight. Bone has been tilted to display features.)

Depth of stylo-mastoid foramen (37 bones valid) : Highest, $17 \mathrm{~mm}$. ; lowest, $7 \mathrm{~mm}$.; average, $11.6 \mathrm{~mm}$.

(Note.-This last measurement was only recorded when the canal could be projected on to the mastoid process. When it lay further forward so as to correspond to the meatus the measurement was not taken.)

In the first decade these distances were: Upper end of verticalhighest, $14 \mathrm{~mm}$.; lowest, $3.5 \mathrm{~mm}$.; average, $8.8 \mathrm{~mm}$.; and stylo-mastoid foramen-highest, $10 \mathrm{~mm}$.; lowest, $5 \mathrm{~mm}$.; average, $7.7 \mathrm{~mm}$.

The Pyramidal Segment or "Bend" is that portion of the Fallopian aqueduct which lies medial and slightly posterior to the pyramid, where the canal changes its direction from the approximate horizontal of the tympanic segment to the vertical (see Figs. 14, 16, 19, 21, 34, 35, 36). 
It is thus a rectangular curve, which in most of the specimens was found to be gradual, the curve implicating from 2 to $6 \mathrm{~mm}$. of the canal. In four adult specimens out of 39 bones, however, the angle was abrupt, and the same characterised two of the ten infantile bones (Fig. 33).

Although the sinus tympani comes into relationship with the upper part of the vertical rather than with the pyramidal segment, this is perhaps the most convenient place to deal with that recess, which is a small cell-like extension of the tympanic cavity backwards rather below the level of the oval window extending under the Fallopian canal in such a way as to undercut it.

The practical interest in the sinus consists in the fact that this is a favourite site for caries of the bone, with granulation-tissue formation, in chronic suppuration of the middle ear, and that in performing the radical mastoid operation one frequently finds oneself impelled to curette this region thoroughly (see later). The proximity of the

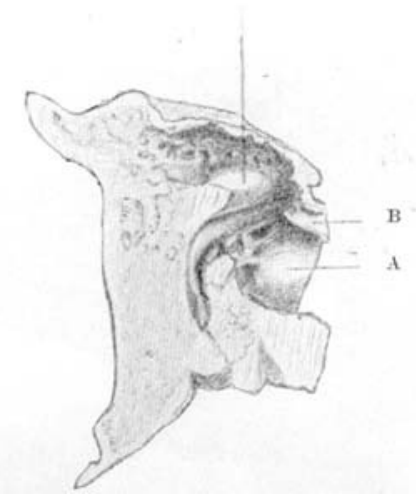

Fig. 19.-The pyramidal (bend) and tympanic segments opened up to show their relationship to the tympanic cavity, oval window, processus cochleariformis (B) and external semicircular canal (C). A is the promontory. The petrous has been divided across transversely. (Bone 36, right temporal, male, aged nine, slightly +, natural pose.)

Fallopian canal renders such a procedure hazardous to the facial nerve. Consequently, it is important to obtain data regarding the recess.

Thirty-five adult bones were examined in this matter; in 13 , the sinus tympani was "shallow" and ill-formed in 21 it was "deep," and in one it seemed to surround the canal, but it did not actually do so, the note on the finding being (Bone No. 42): "Sinus tympani in apposition with a mastoid cell under the canal, but not communicating with that cell." In one (Bone No. 2) it did, however, entirely pass behind the canal to communicate with the mastoid cells, but whether this communication was natural or artificial I cannot say.

In the ten bones of the first decade seven showed a deep undercut by the sinus tympani, one was "shallow," and in one it was absent.

The depth of the sinus tympani ranged from 0.5 to $3 \mathrm{~mm}$.

The development of the sinus tympanicus therefore shows considerable variation, and this is also borne out by the Cheatle collection, in which several specimens show quite considerable cell-like developments 
of this cul de sac. In one it runs "backwards, outwards and downwards behind the facial nerve to below the loop of the external semicircular canal, the inner wall being formed by the vestibule, posterior semicircular canal and sulcus jugularis, where the bone is very thin."

Obviously, then, suppuration and caries of this region must expose the nerve to serious danger.

The presence of a "pyramidal " cell or cells in relation to the bend has already been considered.

The Tympanic Segment (Figs. 5, 6, 7, 9, 11, 12, 14, 16, 18, 19, 21, $23,27,29,31,33$ and 37 ) extends between the genu and the pyramidal segment. Ordinary descriptions to the effect that it runs between the roof and inner wall of the tympanum are inaccurate. The canal does not reach the roof save at its innermost (medial) end, where it leaves the tympanum for the genu (Fig. 16). The antro-tympanic cavity is so shaped and disposed that the facial canal runs through the tympanum

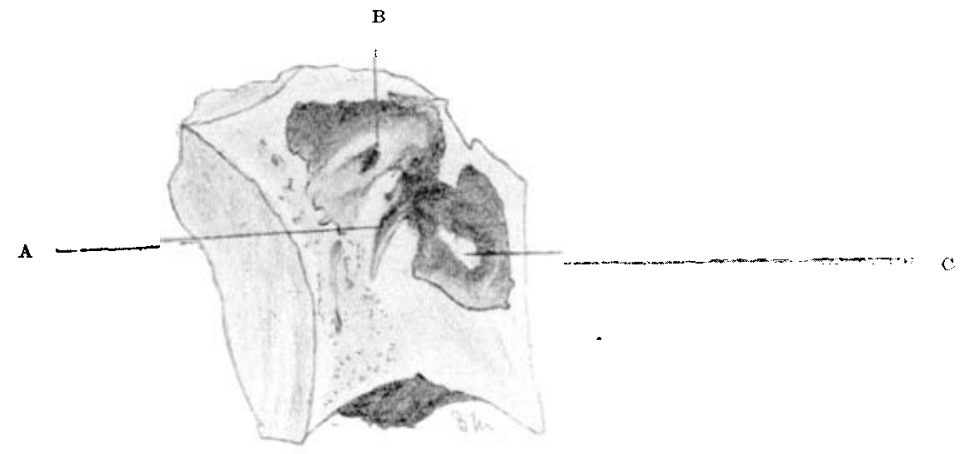

FIG. 20.-Antro-tympanic cavity in an infantile bone from a postero-external position, looking into $\mathrm{c}$, the Eustachian tube. A marks the pyramidal segment of the Fallopian canal, and $B$ is the external semicircular canal opened up to show its relationship to A. (Bone 13, right temporal, slightly + , female, aged seren, pose natural.)

along its posterior internal wall just under the external (lateral) semicircular canal, which bulges out over it. Thus we may say that the aqueduct lies at the junction between the postero-internal tympanic wall and the prominence of the external (lateral) semicircular canal. About midway in its course it comes into close relationship with the oval window and stapes, forming as it does a moulding, so to speak, in the upper arch of the niche. Immediately medial to the oval window it passes behind the nipple-like end of the processus cochleariformis (Fig. 19), and now at length, after having undergone a gradual and steady rise in level as it is followed from without in, it reaches the junction of the roof and inner wall and then straightway leaves the tympanic cavity, piercing the wall to reach the geniculate angle.

Dehiscences in the wall of the tympanic segment are known to occur, and in view of their important clinical bearing considerable attention was devoted to them in the course of the investigation. Out of 42 bones available, dehiscences in the tympanic segment were found as follows: 


\begin{tabular}{|c|c|c|c|c|c|c|}
\hline First & cade & $=$ & & & 0 & $\operatorname{sim}$ \\
\hline Second & ", & $=$ & 0 & , & 4 & ", \\
\hline Third & , & $=$ & 1 & , & 8 & ", \\
\hline Fourth & $"$ & $=$ & 2 & , & 5 & $"$ \\
\hline Fifth & $"$ & $=$ & 2 & ", & 6 & ", \\
\hline Sixth & , & $=$ & 2 & ," & 6 & , \\
\hline Seventh & , & $=$ & 3 & , & 3 & ," \\
\hline
\end{tabular}

That is to say, in specimens from the "first thirty" years of life, only 1 out of 22 bones showed dehiscence, whereas in the last forty years out of 20 bones, 9 , or nearly 50 per cent., showed dehiscences.

This tendency to the appearance of defects in the older bones may presumably be ascribed to the natural osteoporotic changes of advancing

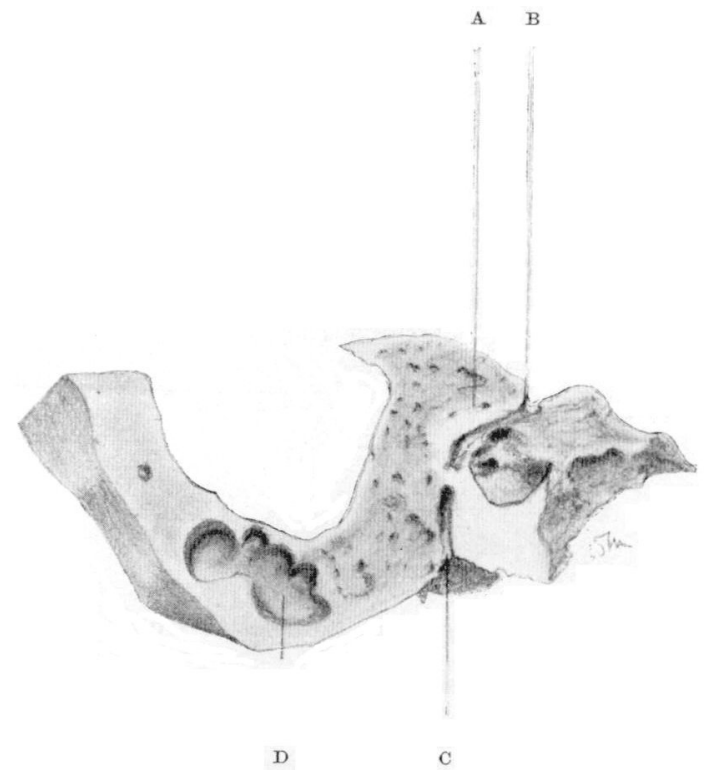

Fia. 21.-Tympanum, aqueduct of Fallopius, and external semicircular canal, A, through which the section has passed. The tympanum has been unroofed. The lower portion of the tympanic membrane is in situ. B. The geniculate bend. c. Stylomastoid foramen. D. A group of large air-cells in close contact with the lateral sinus groove. Note the slope of the tympanic segment and its relations to oval window and external canal (A). (Bone 16, right temporal, male, aged fifty-four, pose natural, slightly reduced.)

years. But it must be recorded that former observers have found dehiscences to be more common in early life.

The extent of the defect varied from an opening one millimetre in length to absence of the whole length of the tympanic bony wall of this segment of the canal.

(It is necessary to add that the condition of the specimens rendered it sometimes difficult to make sure of the presence or absence of bony defects in the tympanic segment. Every effort was made, however, to obtain accurate observation.)

(To be continued.) 